\title{
On some Diophantine equations
}

\author{
Bahar Demirtürk Bitim* and Refik Keskin
}

"Correspondence: demirturk@sakarya.edu.tr Department of Mathematics, Sakarya University, Esentepe Campus, Sakarya, 54187, Turkey

\section{Springer}

\section{Abstract}

We consider the sequences $\left(u_{n}\right)$ and $\left(v_{n}\right)$ which are the generalizations of Fibonacci and Lucas sequences, respectively. Then we determine some identities involving these generalized sequences to present all solutions of the equations

$$
\begin{aligned}
& x^{2}-v_{n} x y+y^{2}=-\left(p^{2}-4\right) u_{n^{\prime}}^{2} \\
& x^{2}-v_{n} x y+y^{2}=u_{n}^{2}
\end{aligned}
$$

and

$$
x^{2}-\left(p^{2}-4\right) u_{n} x y-\left(p^{2}-4\right) y^{2}=v_{n}^{2}
$$

for $p \geq 3$ and a square-free integer $p^{2}-4$. In addition to these, all solutions of some different Diophantine equations such as $x^{2}-v_{2 n} x y+y^{2}=-\left(p^{2}-4\right) u_{n}^{2}$ $x^{2}-v_{n} x y+y^{2}=-\left(p^{2}-4\right), x^{2}-v_{n} x y+y^{2}=1, x^{2}-v_{2 n} x y+y^{2}=u_{n}^{2}, x^{2}-v_{2 n} x y+y^{2}=v_{n}^{2}$ $x^{2}-\left(p^{2}-4\right) u_{n} x y-\left(p^{2}-4\right) y^{2}=1$ are identified, by using divisibility rules of the sequences $\left(u_{n}\right)$ and $\left(v_{n}\right)$.

MSC: 11B37;11B39; 11C20; 11D09; 11D45

Keywords: generalized Fibonacci and Lucas sequences; Diophantine equations

\section{Introduction}

In this paper, we consider the generalized Fibonacci sequence $\left(u_{n}\right)$ and the generalized Lucas sequence $\left(v_{n}\right)$. Let $p \geq 3$ be an integer. For any $n \geq 2,\left(u_{n}\right)$ is defined by the recurrence relation $u_{n}=p u_{n-1}-u_{n-2}$ with the initial conditions $u_{0}=0, u_{1}=1$. The generalized Lucas sequence $\left(v_{n}\right)$ is defined by the recurrence relation $v_{n}=p v_{n-1}-v_{n-2}$ for any $n \geq 2$ with the initial conditions $v_{0}=2$ and $v_{1}=p$. The terms $u_{n}$ and $v_{n}$ are called the $n$th generalized Fibonacci and Lucas numbers, respectively.

Moreover, generalized Fibonacci and Lucas numbers can be extended to negative indices. In general, for all $n \in \mathbb{N}, u_{-n}=-u_{n}$ and $v_{-n}=v_{n}$. Furthermore it is known that $v_{n}=u_{n+1}-u_{n-1}$. For more detailed information about these sequences, one can consult [1-5] and [6].

In [3], McDaniel showed that the solutions of the equation $x^{2}-\left(p^{2}-4\right) y^{2}=4$ are given by $(x, y)=\left(v_{n}, u_{n}\right)$ with $n \geq 1$. Moreover, in [7-9] and [10], Jones investigated whether the equations $x^{2}-\left(p^{2} \mp 4\right) y^{2}=\mp 4, x^{2}-\left(p^{2} \mp 1\right) y^{2}=\mp 4, x^{2}-\left(p^{2} \mp 1\right) y^{2}=\mp 1$ and $x^{2}-\left(p^{2} \mp 4\right) y^{2}=$ $\mp 1$ have solutions or not. In his proofs, he used Fermat's method of infinite descent.

(c) 2013 Demirtürk Bitim and Keskin; licensee Springer. This is an Open Access article distributed under the terms of the Creative Commons Attribution License (http://creativecommons.org/licenses/by/2.0), which permits unrestricted use, distribution, and reproduction in any medium, provided the original work is properly cited. 
In [11], Demirtürk and Keskin determined all solutions of the known Diophantine equations $x^{2}-L_{n} x y-y^{2}=\mp 1, x^{2}-L_{n} x y+(-1)^{n} y^{2}=\mp 5$ and new Diophantine equations

$$
\begin{aligned}
& x^{2}-5 F_{n} x y-5(-1)^{n} y^{2}=\mp 1, \\
& x^{2}-L_{2 n} x y+y^{2}=\mp 5 F_{n}^{2}, \\
& x^{2}-L_{2 n} x y+y^{2}=\mp F_{n}^{2}, \\
& x^{2}-L_{2 n} x y+y^{2}=\mp L_{n}^{2}
\end{aligned}
$$

and

$$
x^{2}-L_{2 n} x y+y^{2}=\mp 5 L_{n}^{2} .
$$

In this paper, our main purpose is to determine all $(x, y)$ solutions of the Diophantine equations

$$
x^{2}+a x y+b y^{2}=c,
$$

where $a, b, c$ are generalized Fibonacci and generalized Lucas numbers. These equations can be listed as follows:

$$
\begin{aligned}
& x^{2}-v_{n} x y+y^{2}=-\left(p^{2}-4\right) u_{n}^{2}, \\
& x^{2}-v_{n} x y+y^{2}=u_{n}^{2}, \\
& x^{2}-\left(p^{2}-4\right) u_{n} x y-\left(p^{2}-4\right) y^{2}=v_{n}^{2}, \\
& x^{2}-v_{2 n} x y+y^{2}=-\left(p^{2}-4\right) u_{n}^{2}, \\
& x^{2}-v_{n} x y+y^{2}=-\left(p^{2}-4\right), \\
& x^{2}-v_{n} x y-y^{2}=1, \\
& x^{2}-v_{2 n} x y+y^{2}=u_{n}^{2}, \\
& x^{2}-v_{2 n} x y+y^{2}=v_{n}^{2}
\end{aligned}
$$

and

$$
x^{2}-\left(p^{2}-4\right) u_{n} x y-\left(p^{2}-4\right) y^{2}=1 .
$$

\section{Divisibility rules of sequences $\left(u_{n}\right)$ and $\left(v_{n}\right)$}

In this section, we recall some divisibility rules related to generalized Fibonacci and Lucas sequences $\left(u_{n}\right)$ and $\left(v_{n}\right)$. Since these rules are proved in [12-16], we omit their proofs. Using these divisibility rules, in the last section, we will find all solutions of Diophantine equations mentioned above.

Theorem 1 Let $m, n \in \mathbb{N}$. Then $v_{n} \mid u_{m}$ if and only if $n \mid m$ and $m / n$ is an even integer.

Theorem 2 Let $m, n \in \mathbb{N}$. Then $u_{n} \mid u_{m}$ if and only if $n \mid m$. 
Theorem 3 Let $m, n \in \mathbb{N}$. Then $v_{n} \mid v_{m}$ if and only if $n \mid m$ and $m / n$ is an odd integer.

Theorem 4 Let $m, n \in \mathbb{N}$ and $n>1$. Then $u_{n} \mid v_{m}$ if and only if $n=2$ and $m$ is an odd integer.

\section{Some identities of the sequences $\left(u_{n}\right)$ and $\left(v_{n}\right)$}

In this section, we obtain some identities by using special matrices including generalized Fibonacci and Lucas numbers.

Now we compile some identities to use in the proofs of the following theorems. These identities can be found in $[5,17,18]$ and $[19]$.

$$
\begin{aligned}
& u_{n}^{2}-p u_{n} u_{n-1}+u_{n-1}^{2}=1, \\
& v_{n}^{2}-p v_{n} v_{n-1}+v_{n-1}^{2}=-\left(p^{2}-4\right), \\
& v_{m} u_{n}-u_{m} v_{n}=2 u_{n-m}, \\
& v_{m} v_{n}-\left(p^{2}-4\right) u_{m} u_{n}=2 v_{n-m}, \\
& u_{n} u_{m+1}-u_{m} u_{n-1}=u_{n+m}, \\
& v_{m} v_{n}+\left(p^{2}-4\right) u_{m} u_{n}=2 v_{n+m}, \\
& u_{m} v_{n}+v_{m} u_{n}=2 u_{n+m}, \\
& u_{n+1}-u_{n-1}=v_{n}, \\
& v_{n+1}-v_{n-1}=\left(p^{2}-4\right) u_{n}, \\
& v_{n}^{2}-\left(p^{2}-4\right) u_{n}^{2}=4 \\
& u_{m+1} v_{n}-u_{m} v_{n-1}=v_{n+m}
\end{aligned}
$$

for all $m, n \in \mathbb{Z}$.

\section{Theorem 5}

$$
v_{n+m}^{2}-\left(p^{2}-4\right) u_{n-t} v_{n+m} u_{m+t}-\left(p^{2}-4\right) u_{m+t}^{2}=v_{n-t}^{2},
$$

for all $m, n, t \in \mathbb{Z}$.

Proof If we consider identities (3.6) and (3.7), then the matrix multiplication

$$
\left[\begin{array}{cc}
v_{n} / 2 & \left(p^{2}-4\right) u_{n} / 2 \\
u_{t} / 2 & v_{t} / 2
\end{array}\right]\left[\begin{array}{l}
v_{m} \\
u_{m}
\end{array}\right]=\left[\begin{array}{l}
v_{n+m} \\
u_{m+t}
\end{array}\right]
$$

can be written. By identity (3.4), we get

$$
\left[\begin{array}{l}
v_{m} \\
u_{m}
\end{array}\right]=\left[\begin{array}{cc}
v_{n} / 2 & \left(p^{2}-4\right) u_{n} / 2 \\
u_{t} / 2 & v_{t} / 2
\end{array}\right]^{-1}\left[\begin{array}{l}
v_{n+m} \\
u_{m+t}
\end{array}\right]=\frac{2}{v_{n-t}}\left[\begin{array}{cc}
v_{t} / 2 & -\left(p^{2}-4\right) u_{n} / 2 \\
-u_{t} / 2 & v_{n} / 2
\end{array}\right]\left[\begin{array}{l}
v_{n+m} \\
u_{m+t}
\end{array}\right],
$$

since

$$
\left|\begin{array}{cc}
v_{n} / 2 & \left(p^{2}-4\right) u_{n} / 2 \\
u_{t} / 2 & v_{t} / 2
\end{array}\right|=\frac{v_{n} v_{t}-\left(p^{2}-4\right) u_{n} u_{t}}{4}=\frac{v_{n-t}}{2} \neq 0
$$


Thus, it follows that

$$
v_{m}=\frac{v_{t} v_{n+m}-\left(p^{2}-4\right) u_{n} u_{m+t}}{v_{n-t}}
$$

and

$$
u_{m}=\frac{v_{n} u_{m+t}-u_{t} v_{n+m}}{v_{n-t}} .
$$

Since $v_{m}^{2}-\left(p^{2}-4\right) u_{m}^{2}=4$, we have

$$
\left(v_{t} v_{n+m}-\left(p^{2}-4\right) u_{n} u_{m+t}\right)^{2}-\left(p^{2}-4\right)\left(v_{n} u_{m+t}-u_{t} v_{n+m}\right)^{2}=4 v_{n-t}^{2} .
$$

Therefore, we obtain $\left(v_{t}^{2}-\left(p^{2}-4\right) u_{t}^{2}\right) v_{n+m}^{2}-2\left(p^{2}-4\right)\left(v_{t} u_{n}-v_{n} u_{t}\right) v_{n+m} u_{m+t}-\left(p^{2}-4\right)\left(v_{n}^{2}-\right.$ $\left.\left(p^{2}-4\right) u_{n}^{2}\right) u_{m+t}^{2}=4 v_{n-t}^{2}$. By using (3.3) and (3.10) in this equation, it is seen that

$$
4 v_{n+m}^{2}-4\left(p^{2}-4\right) u_{n-t} v_{n+m} u_{m+t}-4\left(p^{2}-4\right) u_{m+t}^{2}=4 v_{n-t}^{2}
$$

Thus, we get

$$
v_{n+m}^{2}-\left(p^{2}-4\right) u_{n-t} v_{n+m} u_{m+t}-\left(p^{2}-4\right) u_{m+t}^{2}=v_{n-t}^{2} .
$$

Theorem 6 Let $m, n, t \in \mathbb{Z}$ and $t \neq n$. Then

$$
v_{n+m}^{2}-v_{n-t} v_{n+m} v_{m+t}+v_{m+t}^{2}=-\left(p^{2}-4\right) u_{n-t}^{2} .
$$

Proof By using (3.6), we can consider the matrix multiplication

$$
\left[\begin{array}{ll}
v_{n} / 2 & \left(p^{2}-4\right) u_{n} / 2 \\
v_{t} / 2 & \left(p^{2}-4\right) u_{t} / 2
\end{array}\right]\left[\begin{array}{l}
v_{m} \\
u_{m}
\end{array}\right]=\left[\begin{array}{l}
v_{n+m} \\
v_{m+t}
\end{array}\right]
$$

Since $t \neq n$, we get

$$
\left|\begin{array}{ll}
v_{n} / 2 & \left(p^{2}-4\right) u_{n} / 2 \\
v_{t} / 2 & \left(p^{2}-4\right) u_{t} / 2
\end{array}\right|=\frac{\left(p^{2}-4\right)\left(v_{n} u_{t}-v_{t} u_{n}\right)}{4}=\frac{\left(p^{2}-4\right) u_{n-t}}{2} \neq 0
$$

by (3.3). Therefore, we have

$$
\begin{aligned}
{\left[\begin{array}{l}
v_{m} \\
u_{m}
\end{array}\right] } & =\left[\begin{array}{ll}
v_{n} / 2 & \left(p^{2}-4\right) u_{n} / 2 \\
v_{t} / 2 & \left(p^{2}-4\right) u_{t} / 2
\end{array}\right]^{-1}\left[\begin{array}{c}
v_{n+m} \\
v_{m+t}
\end{array}\right] \\
& =\frac{2}{\left(p^{2}-4\right) u_{n-t}}\left[\begin{array}{cc}
\left(p^{2}-4\right) u_{t} / 2 & -\left(p^{2}-4\right) u_{n} / 2 \\
-v_{t} / 2 & v_{n} / 2
\end{array}\right]\left[\begin{array}{c}
v_{n+m} \\
v_{m+t}
\end{array}\right] .
\end{aligned}
$$

Thus, it follows that

$$
v_{m}=\frac{u_{t} v_{n+m}-u_{n} v_{m+t}}{u_{n-t}}
$$


and

$$
u_{m}=\frac{v_{n} v_{m+t}-v_{t} v_{n+m}}{\left(p^{2}-4\right) u_{n-t}} .
$$

Since $v_{m}^{2}-\left(p^{2}-4\right) u_{m}^{2}=4$, we get

$$
\left(p^{2}-4\right)\left(u_{t} v_{n+m}-u_{n} v_{m+t}\right)^{2}-\left(v_{n} v_{m+t}-v_{t} v_{n+m}\right)^{2}=4\left(p^{2}-4\right) u_{n-t}^{2}
$$

Hence, it is seen that $-\left(v_{t}^{2}-\left(p^{2}-4\right) u_{t}^{2}\right) v_{n+m}^{2}+2\left(v_{n} v_{t}-\left(p^{2}-4\right) u_{t} u_{n}\right) v_{n+m} v_{m+t}-\left(v_{n}^{2}-\left(p^{2}-\right.\right.$ 4) $\left.u_{n}^{2}\right) v_{m+t}^{2}=4\left(p^{2}-4\right) u_{n-t}^{2}$. By using identities (3.4) and (3.10), we obtain

$$
4 v_{n+m}^{2}-4 v_{n-t} v_{n+m} v_{m+t}+4 v_{m+t}^{2}=-4\left(p^{2}-4\right) u_{n-t}^{2},
$$

that is,

$$
v_{n+m}^{2}-v_{n-t} v_{n+m} v_{m+t}+v_{m+t}^{2}=-\left(p^{2}-4\right) u_{n-t}^{2} .
$$

Using (3.7) and the matrix multiplication

$$
\left[\begin{array}{ll}
u_{n} / 2 & v_{n} / 2 \\
u_{t} / 2 & v_{t} / 2
\end{array}\right]\left[\begin{array}{l}
v_{m} \\
u_{m}
\end{array}\right]=\left[\begin{array}{l}
u_{n+m} \\
u_{m+t}
\end{array}\right],
$$

we can give the following theorem.

Theorem 7 Let $m, n, t \in \mathbb{Z}$ and $t \neq n$. Then

$$
u_{n+m}^{2}-v_{n-t} u_{n+m} u_{m+t}+u_{m+t}^{2}=u_{n-t}^{2} .
$$

\section{Solutions of some Diophantine equations}

In [4], Melham proved that all solutions of the equations $y^{2}-v_{m} x y+x^{2}=\mp u_{m}^{2}$ are $(x, y)=$ $\mp\left(u_{n}, u_{n+m}\right)$ with $n \in \mathbb{Z}$. Moreover, he showed that if $m \in \mathbb{Z}$ and $p^{2}-4$ is a square-free integer, then all solutions of the equation $y^{2}-v_{m} x y+x^{2}=-\left(p^{2}-4\right) u_{m}^{2}$ are given by $(x, y)=$ $\mp\left(v_{n}, v_{n+m}\right)$ with $n \in \mathbb{Z}$. These theorems of Melham are generalized forms of the theorems given in [3], by McDaniel. In [2], Kılıç and Ömür examined more general situations of the conics that McDaniel and Melham dealt in [3] and [4], respectively.

In this section, using the identities given in (3.13) and (3.14), we will obtain all solutions of the equations

$$
x^{2}-v_{n} x y+y^{2}=-\left(p^{2}-4\right) u_{n}^{2}
$$

and

$$
x^{2}-v_{n} x y+y^{2}=u_{n}^{2}
$$

with $n \geq 1, p \geq 3$ and $p^{2}-4$ is a square-free integer. The solutions of these equations were explored by Kimberling, McDaniel and Melham, respectively in [3, 20] and [4], but we will 
give different proofs of them here. Moreover, for $p \geq 3$, we will obtain all solutions of the Diophantine equation

$$
x^{2}-\left(p^{2}-4\right) u_{n} x y-\left(p^{2}-4\right) y^{2}=v_{n}^{2},
$$

by using (3.12). Subsequently, if $p \geq 3$ and $p^{2}-4$ is a square-free integer, then we will find all solutions of Diophantine equations

$$
x^{2}-v_{2 n} x y+y^{2}=-\left(p^{2}-4\right) u_{n}^{2}
$$

and

$$
x^{2}-v_{n} x y+y^{2}=-\left(p^{2}-4\right) .
$$

Moreover, all solutions of the equations

$$
x^{2}-v_{n} x y+y^{2}=1, \quad x^{2}-v_{2 n} x y+y^{2}=u_{n}^{2}
$$

and

$$
x^{2}-v_{2 n} x y+y^{2}=v_{n}^{2}
$$

will be determined. Addition to this, if $p \geq 3$, then all solutions of the equation

$$
x^{2}-\left(p^{2}-4\right) u_{n} x y-\left(p^{2}-4\right) y^{2}=1
$$

will be found.

Now we will remind some Diophantine equations with their solutions. The solutions of these equations are explored in [3] and [6]. We will use these equations for determining all solutions of other Diophantine equations.

Theorem 8 Let $p>3$. All solutions of the equation $x^{2}-p x y+y^{2}=1$ are given by $(x, y)=$ $\mp\left(u_{m}, u_{m-1}\right)$ with $m \in \mathbb{Z}$.

Since Corollary 1 can be seen from Theorem 8 and Corollary 2 is stated in [10], we will give them without proof.

Corollary 1 All solutions of the equation $x^{2}-3 x y+y^{2}=1$ are given by $(x, y)=\mp\left(F_{2 m+2}, F_{2 m}\right)$ with $m \in \mathbb{Z}$.

Corollary 2 Let $p>3$. All nonnegative solutions of the equation $u^{2}-\left(p^{2}-4\right) v^{2}=4$ are given by $(u, v)=\left(v_{m}, u_{m}\right)$ with $m \geq 1$.

Theorem 9 and Theorem 10 are stated in [21], so will give them without proof.

Theorem 9 Let $p>3$. Then the equation $x^{2}-p x y+y^{2}=-1$ has no solutions.

Theorem 10 All solutions of the equation $x^{2}-3 x y+y^{2}=-1$ are given by $(x, y)=$ $\mp\left(F_{2 m+1}, F_{2 m-1}\right)$ with $m \in \mathbb{Z}$. 
From now on we will assume that $n$ is an integer such that $n \geq 1$.

Theorem 11 If $p \geq 3$, then all solutions of the equation $x^{2}-\left(p^{2}-4\right) u_{n} x y-\left(p^{2}-4\right) y^{2}=v_{n}^{2}$ are given by $(x, y)=\mp\left(v_{n+m}, u_{m}\right)$ with $m \in \mathbb{Z}$.

Proof Assume that $x^{2}-\left(p^{2}-4\right) u_{n} x y-\left(p^{2}-4\right) y^{2}=v_{n}^{2}$ for some integers $x$ and $y$. Hence, we can write

$$
\left(2 x-\left(p^{2}-4\right) u_{n} y\right)^{2}-\left(\left(p^{2}-4\right)^{2} u_{n}^{2}+4\left(p^{2}-4\right)\right) y^{2}=4 v_{n}^{2} .
$$

Thus, it follows that $\left(2 x-\left(p^{2}-4\right) u_{n} y\right)^{2}-\left(p^{2}-4\right)\left(\left(p^{2}-4\right) u_{n}^{2}+4\right) y^{2}=4 v_{n}^{2}$. By using (3.10) in this equation, we get $\left(2 x-\left(p^{2}-4\right) u_{n} y\right)^{2}-\left(p^{2}-4\right) v_{n}^{2} y^{2}=4 v_{n}^{2}$. Therefore it can be seen that $v_{n} \mid 2 x-\left(p^{2}-4\right) u_{n} y$. Then taking

$$
u=\frac{\left(\frac{\left(2 x-\left(p^{2}-4\right) u_{n} y\right)}{v_{n}}+p y\right)}{2} \text { and } v=y
$$

we obtain $u=\left(x+v_{n-1} y\right) / v_{n}$, by (3.9). From here we get

$$
\begin{aligned}
u^{2}-p u v+v^{2} & =\left(\left(x+v_{n-1} y\right) / v_{n}\right)^{2}-p\left(\left(x-v_{n-1} y\right) / v_{n}\right) y+y^{2} \\
& =\left(x^{2}-\left(v_{n+1}-v_{n-1}\right) x y+y^{2}\left(v_{n}^{2}-p v_{n} v_{n-1}+v_{n-1}^{2}\right)\right) / v_{n}^{2} .
\end{aligned}
$$

Hence, it follows that

$$
u^{2}-p u v+v^{2}=\left(x^{2}-\left(p^{2}-4\right) u_{n} x y-\left(p^{2}-4\right) y^{2}\right) / v_{n}^{2}=v_{n}^{2} / v_{n}^{2}=1,
$$

by using (3.2) and (3.9). From Theorem 8 , we obtain $(u, v)=\mp\left(u_{m+1}, u_{m}\right)$ for some $m \in \mathbb{Z}$. Thus, it is seen that

$$
\left(x+v_{n-1} y\right) / v_{n}=\mp u_{m+1} \quad \text { and } \quad y=\mp u_{m}
$$

so we get $x=\mp\left(u_{m+1} v_{n}-v_{n-1} u_{m}\right)$ and $y=\mp u_{m}$. Now using (3.11), we obtain

$$
(x, y)=\mp\left(v_{n+m}, u_{m}\right) .
$$

Conversely, if $(x, y)=\mp\left(v_{n+m}, u_{m}\right)$ with $m \in \mathbb{Z}$, then it can be seen that $x^{2}-\left(p^{2}-4\right) u_{n} x y-$ $\left(p^{2}-4\right) y^{2}=v_{n}^{2}$, by $(3.12)$.

Using Theorem 9 in the same manner with Theorem 11, the following corollary can be given.

Corollary 3 If $p>3$, then the equation $x^{2}-\left(p^{2}-4\right) u_{n} x y-\left(p^{2}-4\right) y^{2}=-v_{n}^{2}$ has no solutions.

Proof Assume that $x^{2}-\left(p^{2}-4\right) u_{n} x y-\left(p^{2}-4\right) y^{2}=-v_{n}^{2}$ for some integers $x$ and $y$. Similar with the proof of Theorem 11, taking $u=\left(x+v_{n-1} y\right) / v_{n}$ and $v=y$, it can be seen that

$$
u^{2}-p u v+v^{2}=\left(x^{2}-\left(p^{2}-4\right) u_{n} x y-\left(p^{2}-4\right) y^{2}\right) / v_{n}^{2}=-v_{n}^{2} / v_{n}^{2}=-1,
$$


which is impossible by Theorem 9. Thus, it follows that the equation $x^{2}-\left(p^{2}-4\right) u_{n} x y-$ $\left(p^{2}-4\right) y^{2}=-v_{n}^{2}$ has no integer solutions.

The following corollary is a result of Theorem 11. Since it is proved in [11], we will give it without proof.

Corollary 4 All solutions of the equation $x^{2}-5 F_{2 n} x y-5 y^{2}=-L_{2 n}^{2}$ are given by $(x, y)=$ $\mp\left(L_{2 n+2 m+1}, F_{2 m+1}\right)$ with $m \in \mathbb{Z}$.

Theorem 12 and Theorem 13 are stated by Melham, Kılıç and Ömür without proof in [4] and [2], respectively. Now we will prove them.

Theorem 12 Let $p \geq 3$ and $p^{2}-4$ be a square-free integer. Then all solutions of the equation $x^{2}-v_{n} x y+y^{2}=-\left(p^{2}-4\right) u_{n}^{2}$ are given by $(x, y)=\mp\left(v_{n+m}, v_{m}\right)$ with $m \in \mathbb{Z}$.

Proof Assume that $x^{2}-v_{n} x y+y^{2}=-\left(p^{2}-4\right) u_{n}^{2}$ for some integers $x$ and $y$. Then multiplying both sides of this equation by 4 and using (3.10), we get $\left(2 x-v_{n} y\right)^{2}-\left(p^{2}-4\right) u_{n}^{2} y^{2}=-4\left(p^{2}-\right.$ 4) $u_{n}^{2}$. Since $p^{2}-4$ is square-free, it follows that $u_{n} \mid 2 x-v_{n} y$. Therefore, there is an integer $z$ such that $2 x-v_{n} y=u_{n} z$. From here we get $\left(u_{n} z\right)^{2}-\left(p^{2}-4\right) u_{n}^{2} y^{2}=-4\left(p^{2}-4\right) u_{n}^{2}$, and then $z^{2}-\left(p^{2}-4\right) y^{2}=-4\left(p^{2}-4\right)$. This implies that $\left(p^{2}-4\right) \mid z$. Then there is an integer $a$ such that $z=\left(p^{2}-4\right) a$, and we have $2 x-v_{n} y=\left(p^{2}-4\right) u_{n} a$. Thus, it follows that

$$
y^{2}-\left(p^{2}-4\right) a^{2}=4
$$

Since

$$
y^{2}-p^{2} a^{2}=4+4 a^{2}
$$

we have $y^{2}-p^{2} a^{2}$ is even. Then we can see that $y$ and $p a$ have the same parity. Taking $u=(y+p a) / 2$ and $v=a$, we obtain

$$
u=\frac{y+p\left(\frac{2 x-v_{n} y}{\left(p^{2}-4\right) u_{n}}\right)}{2}=\frac{2 p x+\left(\left(p^{2}-4\right) u_{n}-p v_{n}\right) y}{2\left(p^{2}-4\right) u_{n}}=\frac{p x-v_{n-1} y}{\left(p^{2}-4\right) u_{n}}
$$

and

$$
v=\frac{2 x-v_{n} y}{\left(p^{2}-4\right) u_{n}} .
$$

Hence, we get

$$
\begin{aligned}
u^{2}-p u v+v^{2} & =\left(\frac{p x-v_{n-1} y}{\left(p^{2}-4\right) u_{n}}\right)^{2}-p\left(\frac{p x-v_{n-1} y}{\left(p^{2}-4\right) u_{n}}\right)\left(\frac{2 x-v_{n} y}{\left(p^{2}-4\right) u_{n}}\right)+\left(\frac{2 x-v_{n} y}{\left(p^{2}-4\right) u_{n}}\right)^{2} \\
& =-\left(p^{2}-4\right)\left(x^{2}-v_{n} x y+y^{2}\right) /\left(p^{2}-4\right)^{2} u_{n}^{2}=1 .
\end{aligned}
$$

Therefore it follows that $(u, v)=\mp\left(u_{m+1}, u_{m}\right)$ with $m \in \mathbb{Z}$ from Theorem 8. Thus, we obtain

$$
\left(p x-v_{n-1} y\right) /\left(p^{2}-4\right) u_{n}=\mp u_{m+1} \quad \text { and } \quad\left(2 x-v_{n} y\right) /\left(p^{2}-4\right) u_{n}=\mp u_{m} .
$$

Using the identities (3.6), (3.8) and (3.9) in (4.1), we get $(x, y)=\mp\left(v_{n+m}, v_{m}\right)$. 
Conversely, if $(x, y)=\mp\left(v_{n+m}, v_{m}\right)$, then it follows that $x^{2}-v_{n} x y+y^{2}=-\left(p^{2}-4\right) u_{n}^{2}$, by (3.13).

Using Theorem 9 in the same manner with Theorem 12, we can give the following corollary.

Corollary 5 Let $p>3$ and $p^{2}-4$ be a square-free integer. Then the equation $x^{2}-v_{n} x y+y^{2}=$ $\left(p^{2}-4\right) u_{n}^{2}$ has no solutions.

We can give the following corollary from Corollary 5.

Corollary 6 Let $p>3$ and $p^{2}-4$ be a square-free integer. Then the equation $x^{2}-v_{n} x y+y^{2}=$ $\left(p^{2}-4\right)$ has no solutions.

When $p=3$, the equation $x^{2}-v_{n} x y+y^{2}=\left(p^{2}-4\right) u_{n}^{2}$ has solutions. In this case we have the equation $x^{2}-L_{2 n} x y+y^{2}=5 F_{2 n}^{2}$. Now we can give all solutions of these equations in the following lemma. Since this lemma is proved in [11], we will give it without proof.

Lemma 1 All solutions of the equation $x^{2}-L_{2 n} x y+y^{2}=5 F_{2 n}^{2}$ are given by $(x, y)=$ $\mp\left(L_{2 n+2 m+1}, L_{2 m+1}\right)$ with $m \in \mathbb{Z}$.

Theorem 13 All solutions of the equation $x^{2}-v_{n} x y+y^{2}=u_{n}^{2}$ are given by $(x, y)=$ $\mp\left(u_{n+m}, u_{m}\right)$ with $m \in \mathbb{Z}$.

Proof Suppose that $x^{2}-v_{n} x y+y^{2}=u_{n}^{2}$ for some integers $x$ and $y$. Completing the square gives $\left(2 x-v_{n} y\right)^{2}-\left(p^{2}-4\right) u_{n}^{2} y^{2}=4 u_{n}^{2}$, and it is seen that $u_{n} \mid 2 x-v_{n} y$. Thus, it follows that

$$
\left(\left(2 x-v_{n} y\right) / u_{n}\right)^{2}-\left(p^{2}-4\right) y^{2}=4
$$

Taking $u=\left(\left(\left(2 x-v_{n} y\right) / u_{n}\right)+p y\right) / 2=\left(x+u_{n-1} y\right) / u_{n}$ and $v=y$, we have $u^{2}-p u v+v^{2}=1$. Therefore, from Theorem 8 , we get $(u, v)=\mp\left(u_{m+1}, u_{m}\right)$ with $m \in \mathbb{Z}$. From here, we obtain $(x, y)=\mp\left(u_{n} u_{m+1}-u_{n-1} u_{m}, u_{m}\right)$. Then by (3.5), it follows that $(x, y)=\mp\left(u_{n+m}, u_{m}\right)$.

Conversely, if $(x, y)=\mp\left(u_{n+m}, u_{m}\right)$, then it can be seen that $x^{2}-v_{n} x y+y^{2}=u_{n}^{2}$, by (3.14).

Using Theorem 9 in the same manner with Theorem 13, we can give the following corollaries.

Corollary 7 The equation $x^{2}-v_{n} x y+y^{2}=-u_{n}^{2}$ has no solutions.

The following corollary is a generalized form of Theorem 10. Since it is proved in [11], we will give it without proof.

Corollary 8 All solutions of the equation $x^{2}-L_{2 n} x y+y^{2}=-F_{2 n}^{2}$ are given by $(x, y)=$ $\mp\left(F_{2 n+2 m+1}, F_{2 m+1}\right)$ with $m \in \mathbb{Z}$.

Now, let us examine all solutions of the following equations by using Diophantine equations given in Theorem 11, Theorem 12, Theorem 13 and the divisibility rules of the sequences $\left(u_{n}\right)$ and $\left(v_{n}\right)$. 
Theorem 14 All solutions of the equation $x^{2}-v_{n} x y+y^{2}=1$ are given by $(x, y)=\mp\left(u_{(t+1) n} /\right.$ $\left.u_{n}, u_{t n} / u_{n}\right)$ with $t \in \mathbb{Z}$.

Proof Assume that $x^{2}-v_{n} x y+y^{2}=1$ for some integers $x$ and $y$. Multiplying both sides of this equation by $u_{n}^{2}$, we get

$$
\left(u_{n} x\right)^{2}-v_{n}\left(u_{n} x\right)\left(u_{n} y\right)+\left(u_{n} y\right)^{2}=u_{n}^{2} .
$$

From Theorem 13, it follows that $u_{n} x=\mp u_{n+m}$ and $u_{n} y=\mp u_{m}$ for some integer $m$. Hence, we get $x=\mp u_{n+m} / u_{n}$ and $y=\mp u_{m} / u_{n}$. Since $x$ and $y$ are integers, it is clear that $n \mid m$. Therefore, it follows that $m=t n$ for some $t \in \mathbb{Z}$. Then we obtain

$$
(x, y)=\mp\left(u_{(t+1) n} / u_{n}, u_{t n} / u_{n}\right) .
$$

Conversely, if $(x, y)=\mp\left(u_{(t+1) n} / u_{n}, u_{t n} / u_{n}\right)$ with $t \in \mathbb{Z}$, then it follows that $x^{2}-v_{n} x y+y^{2}=1$, by (3.14).

Multiplying both sides of the equation $x^{2}-v_{n} x y+y^{2}=-1$ by $u_{n}^{2}$ and using Corollary 7 , the following corollary can be given.

Corollary 9 The equation $x^{2}-v_{n} x y+y^{2}=-1$ has no solutions.

Theorem 15 If $p \geq 3$, then all solutions of the equation $x^{2}-\left(p^{2}-4\right) u_{n} x y-\left(p^{2}-4\right) y^{2}=1$ are given by $(x, y)=\mp\left(v_{(2 t+1) n} / v_{n}, u_{2 t n} / v_{n}\right)$ with $t \in \mathbb{Z}$.

Proof Assume that $x^{2}-\left(p^{2}-4\right) u_{n} x y-\left(p^{2}-4\right) y^{2}=1$ for some integers $x$ and $y$. Multiplying both sides of this equation by $v_{n}^{2}$, we get

$$
\left(v_{n} x\right)^{2}-\left(p^{2}-4\right) u_{n}\left(v_{n} x\right)\left(v_{n} y\right)-\left(p^{2}-4\right)\left(v_{n} y\right)^{2}=v_{n}^{2} .
$$

Thus, it follows that $v_{n} x=\mp v_{n+m}$ and $u_{n} y=\mp u_{m}$ according to Theorem 11. Hence, we get $(x, y)=\mp\left(v_{n+m} / v_{n}, u_{m} / v_{n}\right)$. From Theorem 1 and Theorem 3, it can be seen that $n \mid m$ and $m / n$ is an even integer. This implies that $m=2 t n$ for some $t \in \mathbb{Z}$. Therefore, we obtain $(x, y)=\mp\left(v_{(2 t+1) n} / v_{n}, u_{2 t n} / v_{n}\right)$.

Conversely, if $(x, y)=\mp\left(v_{(2 t+1) n} / v_{n}, u_{2 t n} / v_{n}\right)$ for some $t \in \mathbb{Z}$, then it follows that $x^{2}-\left(p^{2}-\right.$ 4) $u_{n} x y-\left(p^{2}-4\right) y^{2}=1$, by (3.12).

The following corollary can be given from Corollary 3.

Corollary 10 If $p \geq 3$, then the equation $x^{2}-\left(p^{2}-4\right) u_{n} x y-\left(p^{2}-4\right) y^{2}=-1$ has no solutions.

Theorem 16 If $p \geq 3$ and $p^{2}-4$ is a square-free integer, then all solutions of the equation $x^{2}-v_{2 n} x y+y^{2}=-\left(p^{2}-4\right) u_{n}^{2}$ are given by $(x, y)=\mp\left(v_{(2 t+3) n} / v_{n}, v_{(2 t+1) n} / v_{n}\right)$ with $t \in \mathbb{Z}$.

Proof Suppose that $x^{2}-v_{2 n} x y+y^{2}=-\left(p^{2}-4\right) u_{n}^{2}$ for some integers $x$ and $y$. Multiplying both sides of this equation by $v_{n}^{2}$ and considering the fact that $u_{2 n}=u_{n} v_{n}$, we get

$$
\left(v_{n} x\right)^{2}-v_{2 n}\left(v_{n} x\right)\left(v_{n} y\right)+\left(v_{n} y\right)^{2}=-\left(p^{2}-4\right) u_{2 n}^{2} .
$$


From Theorem 12, it follows that $v_{n} x=\mp v_{2 n+m}$ and $v_{n} y=\mp v_{m}$. Hence we get $(x, y)=$ $\mp\left(v_{2 n+m} / v_{n}, v_{m} / v_{n}\right)$. Moreover, since $x$ and $y$ are integers, it follows that $n \mid m$ and $m / n$ is an odd integer from Theorem 3. Then there is an integer $t$ such that $m=(2 t+1) n$. Therefore, we obtain $(x, y)=\mp\left(v_{(2 t+3) n} / v_{n}, v_{(2 t+1) n} / v_{n}\right)$.

Conversely, if $(x, y)=\mp\left(v_{(2 t+3) n} / v_{n}, v_{(2 t+1) n} / v_{n}\right)$ for some $t \in \mathbb{Z}$, then it follows that $x^{2}-$ $v_{2 n} x y+y^{2}=-\left(p^{2}-4\right) u_{n}^{2}$, by (3.13).

The following corollary can be proved similar to Theorem 16, by using Corollary 5. So, we omit its proof.

Corollary 11 If $p>3$ and $p^{2}-4$ is a square-free integer, then the equation $x^{2}-v_{2 n} x y+y^{2}=$ $\left(p^{2}-4\right) u_{n}^{2}$ has no solutions.

Theorem 17 All solutions of the equations $x^{2}-v_{2 n} x y+y^{2}=u_{n}^{2}$ and $x^{2}-v_{2 n} x y+y^{2}=v_{n}^{2}$ are given by $(x, y)=\mp\left(u_{(2 t+2) n} / v_{n}, u_{2 t n} / v_{n}\right)$ and $(x, y)=\mp\left(u_{(t+2) n} / u_{n}, u_{t n} / u_{n}\right)$ with $t \in \mathbb{Z}$, respectively.

Proof Assume that $x^{2}-v_{2 n} x y+y^{2}=u_{n}^{2}$ for some integers $x$ and $y$. Multiplying both sides of this equation by $v_{n}^{2}$, we get

$$
\left(v_{n} x\right)^{2}-v_{2 n}\left(v_{n} x\right)\left(v_{n} y\right)+\left(v_{n} y\right)^{2}=u_{2 n}^{2} .
$$

Then from Theorem 13, it follows that $(x, y)=\mp\left(u_{2 n+m} / v_{n}, u_{m} / v_{n}\right)$ for some $m \in \mathbb{Z}$. Hence, using Theorem 1 it is seen that $n \mid m$ and $m / n$ is an even integer. Thus, we have $m=2 t n$ for some $t \in \mathbb{Z}$. Therefore, $(x, y)=\mp\left(u_{(2 t+2) n} / v_{n}, u_{2 t n} / v_{n}\right)$.

Conversely, if $(x, y)=\mp\left(u_{(2 t+2) n} / v_{n}, u_{2 t n} / v_{n}\right)$ for some $t \in \mathbb{Z}$, then by (3.14) it follows that $x^{2}-v_{2 n} x y+y^{2}=u_{n}^{2}$.

Now suppose that $x^{2}-v_{2 n} x y+y^{2}=v_{n}^{2}$ for some integers $x$ and $y$. Multiplying both sides of this equation by $u_{n}^{2}$ and considering Theorem 13 , we get $(x, y)=\mp\left(u_{2 n+m} / u_{n}, u_{m} / u_{n}\right)$ for some $m \in \mathbb{Z}$. Furthermore, since $x$ and $y$ are integers, it follows that $n \mid m$ from Theorem 2 . Then we have $m=t n$ for some $t \in \mathbb{Z}$. Thus, we obtain $(x, y)=\mp\left(u_{(t+2) n} / u_{n}, u_{t n} / u_{n}\right)$.

Conversely, if $(x, y)=\mp\left(u_{(t+2) n} / u_{n}, u_{t n} / u_{n}\right)$, then by (3.14) it follows that $x^{2}-v_{2 n} x y+y^{2}=$ $v_{n}^{2}$

The proof of the following corollary is similar to that of Theorem 17, by using Corollary 7. So, we omit its proof.

Corollary 12 The equations $x^{2}-L_{4 n} x y+y^{2}=-F_{2 n}^{2}$ and $x^{2}-L_{4 n} x y+y^{2}=-L_{2 n}^{2}$ have no solutions.

Theorem 18 Let $p \geq 3, p^{2}-4$ be a square-free integer. If $n>2$, then the equation $x^{2}-v_{n} x y+$ $y^{2}=-\left(p^{2}-4\right)$ has no solutions and all solutions of the equation $x^{2}-\left(p^{2}-2\right) x y+y^{2}=-\left(p^{2}-4\right)$ are given by $(x, y)=\mp\left(\frac{v_{2 t+3}}{p}, \frac{v_{2 t+1}}{p}\right)$ with $t \in \mathbb{Z}$.

Proof Assume that $x^{2}-v_{n} x y+y^{2}=-\left(p^{2}-4\right)$ for some integers $x$ and $y$. Multiplying both sides of this equation by $u_{n}^{2}$, we get

$$
\left(u_{n} x\right)^{2}-v_{n}\left(u_{n} x\right)\left(u_{n} y\right)+\left(u_{n} y\right)^{2}=-\left(p^{2}-4\right) u_{n}^{2}
$$


From Theorem 12, it follows that $(x, y)=\mp\left(v_{n+m} / u_{n}, v_{m} / u_{n}\right)$ with $m \in \mathbb{Z}$. If $n>2$, then $u_{n} \nmid v_{m}$ from Theorem 4 . Therefore, the equation $x^{2}-v_{n} x y+y^{2}=-\left(p^{2}-4\right)$ has no solutions. If $n=2$, then we get that all solutions of the equation $x^{2}-\left(p^{2}-2\right) x y+y^{2}=-\left(p^{2}-4\right)$ are given by $(x, y)=\mp\left(v_{m+2} / u_{2}, v_{m} / u_{2}\right)$ with $m \in \mathbb{Z}$. Hence, it is seen that $m$ is an odd integer according to Theorem 4 . Thus, $m=2 t+1$ for some $t \in \mathbb{Z}$. Therefore, it follows that $(x, y)=\mp\left(\frac{\nu_{2 t+3}}{p}, \frac{\nu_{2 t+1}}{p}\right)$.

\section{Competing interests}

The authors declare that they have no competing interests.

\section{Authors' contributions}

All authors contributed equally in the preparation of this article. Both the authors read and approved the final manuscript.

\section{Acknowledgements}

Dedicated to Professor Hari M Srivastava.

Received: 14 December 2012 Accepted: 22 March 2013 Published: 9 April 2013

\section{References}

1. Keskin, R, Demirtürk, B: Solutions of some Diophantine equations using generalized Fibonacci and Lucas sequences. Ars Comb. 110 July (2013)

2. KIlıç, E, Ömür, N: Conics characterizing the generalized Fibonacci and Lucas sequences with indices in arithmetic progressions. Ars Comb. 94, 459-464 (2010)

3. McDaniel, WL: Diophantine representation of Lucas sequences. Fibonacci Q. 33, 58-63 (1995)

4. Melham, R: Conics which characterize certain Lucas sequences. Fibonacci Q. 35, 248-251 (1997)

5. Rabinowitz, S: Algorithmic manipulation of Fibonacci identities. In: Applications of Fibonacci Numbers, vol. 6, pp. 389-408. Kluwer Academic, Dordrect (1996)

6. Zhiwei, S: Singlefold, Diophantine representation of the sequence $u_{0}=0, u_{1}=1$ and $u_{n+2}=m u_{n+1}+u_{n}$. In: Pure and Applied Logic, pp. 97-101. Beijing Univ. Press, Beijing (1992)

7. Jones, JP: Diophantine representation of the Fibonacci numbers. Fibonacci Q. 13, $84-88$ (1975)

8. Jones, JP: Diophantine representation of the Lucas numbers. Fibonacci Q. 14, 134 (1976)

9. Jones, JP, Kiss, P: On points whose coordinates are terms of a linear recurrence. Fibonacci Q. 31, 239-245 (1993)

10. Jones, JP: Representation of solutions of Pell equations using Lucas sequences. EKTF Tud. Közl., Tanulm. Mat. Tud. Köréböl 30, 75-86 (2003)

11. Demirtürk, B, Keskin, R: Integer solutions of some Diophantine equations via Fibonacci and Lucas numbers. J. Integer Seq. 12, 1-14 (2009)

12. Hilton, P, Pedersen, J, Somer, L: On Lucasian numbers. Fibonacci Q. 35, $43-47$ (1997)

13. Hilton, P, Pedersen, J: On generalized Fibonaccian and Lucasian numbers. Math. Gaz. 90(518), 215-222 (2006)

14. Hinkel, DE: An investigation of Lucas Sequences, pp. 1-35. Master Thesis, Arizona University (2007)

15. Ribenboim, P: An algorithm to determine the points with integral coordinates in certain elliptic curves. J. Number Theory 74, 19-38 (1999)

16. Ribenboim, P: My Numbers, My Friends. Springer, New York (2000)

17. Horadam, AF: Basic properties of certain generalized sequences of numbers. Fibonacci Q. 3(3), 161-176 (1965)

18. Ribenboim, P: Square classes of Fibonacci and Lucas numbers. Port. Math. 46(2), 159-175 (1989)

19. Ribenboim, P: The Little Book of Big Primes. Springer, New York (1991)

20. Kimberling, C: Fibonacci hyperbolas. Fibonacci Q. 28, 22-27 (1990)

21. Keskin, R: Solutions of some quadratic Diophantine equations. Comput. Math. Appl. 60, 2225-2230 (2010)

doi:10.1186/1029-242X-2013-162

Cite this article as: Demirtürk Bitim and Keskin: On some Diophantine equations. Journal of Inequalities and Applications 2013 2013:162. 